\title{
The Transition of Primary Care Group Practices to Next Generation Models: Satisfaction of Staff, Clinicians, and Patients
}

\author{
Therese Zink, MD, MPH, John Kralewski, PhD, and Bryan Dowd, PhD
}

Introduction: Restructuring primary care is essential to achieve the triple aim. This case study examines the human factors of extensive redesign on 2 midsized primary care clinics (clinics A and B) in the Midwest United States that are owned by a large health care system. The transition occurred when while the principles for patient-centered medical home were being rolled out nationally, and before the Affordable Care Act.

Methods: After the transition, interviews and discussions were conducted with 5 stakeholder groups: health system leaders, clinic managers, clinicians, nurses, and reception staff. Using a culture assessment instrument, the responses of personnel at clinics A and B were compared with comparison clinics from another health system that had not undergone transition. Patient satisfaction scores are presented.

Results: Clinics A and B were similar in size and staffing. Three human factor themes emerged from interviews: responses to change, professional and personal challenges due to role redefinition, and the importance of communication. The comparison clinics had an equal or higher mean culture scores compared with the transition clinics (A and B). Patient satisfaction in improved in Clinic A.

Conclusions: The transition took more time than expected. Health system leaders underestimated the stress and the role adjustments for clinicians and nurses. Change leaders need to anticipate the challenge of role redefinition until health profession schools graduate trainees with more experience in new models of team-based care. Incorporating experience with team based, interprofessional care into training is essential to properly prepare future health professionals. ( $\mathrm{J}$ Am Board Fam Med 2017;30: 16-24.)

Keywords: Ambulatory Care Facilities, Health Occupations, Health Personnel, Patient Protection and Affordable Care Act, Patient Satisfaction, Patient-Centered Care, Primary Health Care

Restructuring primary care is essential to achieve the triple aim: improving the experience of care, improving the health of populations, and reducing per capita costs of health care. ${ }^{1}$ The transition to a new model is complex, requires upfront resources, and the results vary by setting. ${ }^{2}$ The planning,

This article was externally peer reviewed.

Submitted 8 April 2016; revised 25 August 2016; accepted 6 September 2016.

From the Department of Family Medicine, Wright State University, Dayton, OH (TZ); Division of Health Policy and Management, University of Minnesota, Minneapolis, MN (JK, BD); Medica Research Institute, Minnetonka, MN (JK).

Funding: This research was funded by AHRQ Grant R03 HS22617.

Conflict of interest: none declared.

Corresponding author: Therese Zink, MD, Department of Family Medicine/Boonshoft School of Medicine, Wright State University, 725 University Blvd. \#320, Fairborn, OH 45324 (E-mail: therese.zink@wright.edu). resources, and time required for the transformation of health care delivery from a physician-centric to a team-centric approach are often underestimated. ${ }^{3}$

We had the opportunity to examine the human and financial costs of extensive redesign on 2 midsized primary care clinics in the Midwest United States that are owned by a large health care system with 3 hospitals and thirty clinics. Clinics were directed to transition by health system leadership in 2007 before the principles for patient-centered medical home were being rolled out nationally, and before the Affordable Care Act and the formation of accountable care organizations. Local employers pressured health system leadership to reduce costs and improve value and offered financial help. Clinic A transitioned in the first wave (2008 to 2009) and Clinic B in the second wave (2010 to 2011). Second 
wave clinics adapted the model developed by firstwave clinics, with the latitude to innovate to meet their unique needs. The complete study report is available on the Agency for Health Care Research and Quality web site. ${ }^{4}$ A description and analysis of the new model and financial costs (development time, consultants, information technology support, salary guarantees, lost revenue, and impact of the redesign on claims data) are published elsewhere. ${ }^{5}$ Because payment reform by the major health insurance plan did not occur as quickly as health system leadership anticipated, the new model was scaled back in 2012. In this article we examine the human factors of this transformation on clinicians, staff, and patients based on interviews after the transition, culture assessment results, and patient satisfaction scores.

\section{Methods}

During introductory meetings with health system leadership, we identified 2 clinic managers who were willing to share data and make clinic personnel available for interviews. Clinic A transitioned in the first wave (2008 to 2009) and was fully transformed for 2 years before payment challenges forced scaling back. Clinic B transitioned in the second wave (2010 to 11), made some adaptations to the first wave's model, and experienced a full year of the transition before retrenching.

Our research design was based on standard case study methodology ${ }^{6}$ and our own previous research on medical group practices. ${ }^{7}$ Structured individual interviews were conducted with health system leaders and clinic managers between October 2013 and February 2014. Group discussion over breakfast or lunch were held with clinicians (physicians and nurse practitioners), nurses (RNs, LPNs, and MAs), and reception staff. Physicians and staff who left as a result of the transition were not available for interviews. Two researchers audiotaped the interviews, 1 led the inquiry while the other focused on note taking. Interview questions were developed from the medical home, accountable care organization, and relevant case study literature, ${ }^{2,7-12}$ and reviewed by a primary care physician who was not part of the research team or the health system. Additional questions evolved from some responses. For example, when a nurse reported stress related to expanded duties, we explored the sources of the stress and how the issues were resolved.
Personnel at Clinics A and B completed a validated culture assessment instrument ${ }^{13}$ during our data collection. Scores were compiled and compared with those of personnel at 6 comparison clinics (local, primary care clinics owned by another health system.) The staff and clinicians at the comparison clinics completed the culture instrument for another study before restructuring occurred in their settings. Press Ganey patient experience scores $^{14}$ were available for Clinic A before, during, and after the transition.

The researchers used immersion crystallization techniques to identify human factor themes in the interview data. Disagreements were resolved through discussion until consensus was reached. ${ }^{15}$ Clinic A, Clinic B, and the comparison clinics were compared on the mean score for each of the 10 culture assessment categories. The percent of Clinic A patients who rated their health care experience as satisfactory/very satisfactory is reported for each year.

\section{Results}

As shown in Table 1, the 2 suburban primary care clinics were similar in size. Both employed nurse practitioners, but not physician assistants. Clinic B had fewer registered nurses per provider, probably causing the slightly higher net revenue percentage.

Table 2 presents descriptions of the 32 individuals across the 5 stakeholder groups in Clinics A and $\mathrm{B}$ that we interviewed: health system leaders, managers, clinicians, nurses, and reception staff.

Three human factor themes emerged: responses to change, professional and personal challenges due to role redefinition, and the importance of communication. Each theme is discussed and additional quotes are presented in Table 3.

Responses to change were both taxing and exciting for all stakeholders. One health system leader remarked, "I walked out of the last of 3 planning meetings in the C-suite not believing we could really do this (the directive to transform all the clinics in the health system)." Another leader, a primary care physician, committed to staying in her leadership role 6 months longer than planned in order launch the transition: "My interest was sparked because I am a socialist at heart. As a primary care doc I had seen the inequity—rescuing patients from catastrophic disease instead of preventing illness ... This was a terrific opportunity to craft another way of doing it [providing care].” 
Table 1. Descriptive Information for Clinic A Before (2007), During (2009), and After (2013) the Transition to an Open-Access, Patient-Centered Care Model

\begin{tabular}{|c|c|c|c|c|}
\hline & \multicolumn{3}{|c|}{ Clinic A } & \multirow{2}{*}{$\frac{\text { Clinic B }}{\text { Before Transition }}=$} \\
\hline & $\begin{array}{l}\text { Before Transition } \\
\text { (2007) }\end{array}$ & $\begin{array}{l}\text { During Transition } \\
\text { (2009) }\end{array}$ & $\begin{array}{l}\text { After Transition } \\
\text { (2013) }\end{array}$ & \\
\hline $\begin{array}{l}\text { Full-time equivalent }(\mathrm{FTE})^{*} \text { primary care } \\
\text { physicians }\end{array}$ & 7.9 & 8.5 & 9.3 & 6.6 \\
\hline FTE nurse practitioners & 1.5 & 0.9 & 0.8 & 1.0 \\
\hline FTE support staff & 18.2 & 23.1 & 29.4 & 16.5 \\
\hline Registered nurse & 4.9 & 7.4 & 10.4 & 3 \\
\hline Registered nurse care coordinator & & & 0.6 & \\
\hline Licensed practical nurse /medical assistant & 10.0 & 10.6 & 13.0 & \\
\hline Technician (lab, x-ray) & 4.3 & 5.1 & 5.4 & \\
\hline Total revenue & $\$ 6,297,991$ & $\$ 6,195,565$ & $\$ 8,958,742$ & $6,084,362$ \\
\hline Active patient charts\# & 14,383 & & & 12,340 \\
\hline $\begin{array}{l}\text { Average No. patients/normal work day for } \\
\text { physician/Nurse Practitioner }\end{array}$ & $15.6 / 15.6$ & & $17 / 19$ & \\
\hline \multirow{2}{*}{$\begin{array}{l}\text { Average number of patients/ hour for } \\
\text { physician/Nurse Practitioner }\end{array}$} & $4 / 3$ & $13 / 13$ & $2.2 / 2.5$ & \\
\hline & & $2 / 2$ & & \\
\hline Net revenue per operating costs & $48 \%$ & & & $51 \%$ \\
\hline
\end{tabular}

Description of Clinic B Before the transition (2007). During and after transition data for Clinic B were incomplete.

Specialty physicians and their support staff, pharmacists and social worker are not included.

*1 FTE equals 34 clinical contact hours per week.

\#Active charts includes patients seen in the last two years.

The manager at Clinic A "liked to experiment" and as a result that clinic was chosen to be one of the first to transition. That manager described the responses of clinic personnel: "We needed to understand the incredible toll on staff, we kept asking folks to do more, work longer hours, do homework at night. At first it was exciting, but also stressful. They were working harder, but also enjoying it more."

Some clinicians and staff embraced the changes, others endured and adapted, a few found it too "disruptive." Stakeholders linked the resignation of several physicians to their unwillingness to approach patient care as a team. A clinic manager noted: "Physicians who could not give up control and embrace teamwork left, but others heard about what we were doing, applied, and were hired."

These quotes demonstrate the varied responses: "a terrific opportunity," "the chance to work at the top of your license," "working harder, although enjoying it more," "losing some physicians, but attracting others who were excited about the new model of practice."

In addition to the loss of clinicians, resistance came from some clinical staff and hospitals. For

Table 2. Position Categories for 32 Individuals in Five Stakeholder Groups Interviewed During this Case Study of Clinics A and B

\begin{tabular}{ll}
\hline Stakeholder Group & \multicolumn{1}{c}{ Position Category } \\
\hline Leaders & 3 medical directors, 2 physician leaders at Clinics A and B \\
Managers & 2 quality improvement manager, 2 clinic managers at Clinics A and B \\
Clinicians at Clinics A and B & 4 physicians, 2 nurse practitioners \\
Nurses at Clinics A and B & 5 registered nurses, 3 licensed practical nurses, 6 medical assistants \\
Reception staff at Clinics A and B & 3 front desk/reception staff \\
\hline
\end{tabular}

Individual interviews occurred with leaders (5) and managers (4). Two discussion groups were conducted at each clinic (AM and noon hour). Group sizes ranged from five to six individuals, including a total of 23 clinicians, nurses and reception staff.

Financial personnel were interviewed but data are not included in this count or in the report. 
Table 3. Quotations Illustrating the Human Factors Identified during Interviews or Discussion Groups of the Five Stakeholder Groups in This Case Study

Response to Change

Medical Director Health System leader:

"I walked out of the last of three planning meetings in the C-suite not believing we could really do this."

Medical Director Health System leader:

"In hindsight, we missed the scariness and grief about what we asked them to give up."

Quality Improvement Manager:

"Following change theory, some clinics and people embrace change and others do not. Instead they find it difficult and disruptive. Stress levels of staff were high, for some higher than others. Some providers jumped on board and others refused to collaborate with their teams."

Clinic Manager:

"We needed to understand the incredible toll on staff, we kept asking folks to do more, to work longer hours, do homework at night. At first it was exciting, but also stressful. They were working harder, but also enjoying it more."

Clinic Manager:

"We were cutting costs and the hospital volumes dropped. Hospital administrators got nervous and there was push back."

Clinic Manager:

"Nurses started overseeing self-collected samples for vaginitis. Patients celebrated the end of stirrups, but lab personnel were initially reluctant to accept the specimens."

Clinic Manager:

"Physicians who could not give up control and embrace teamwork left, but others heard about what we were doing, applied, and were hired."

Nurse:

"At one point I felt like we were jumping off a cliff. At night I studied about anti-hypertensive drugs. I needed to understand them if I was responsible for suggesting medication changes to patients. Of course, I could ask for help, but it was very stressful."

Quality Improvement Manager:

"One of our biggest learnings was that we need to match trajectory of change to business model and keep in synch.

Corporate moved too fast. In our market, our payer and us were the first to do this. We still couldn't bill for our

population health or e-visits. We had to scale back. The back paddling was tough as well."

Personal and Professional challenge due to role redefinition:

Physician Health System Leader:

"The most successful clinics are those that embraced the team-based model—gave up own work to a care team and trusted staff. Practices where doctors own their results and can't share the work with the team still complain about WAC [work after clinic]."

Physician leader:

"The solo hero western model that embraces 'I am your doctor' had to change to 'I am your doctor and my team includes ..."

Physician:

"I liked leaving the blood pressure med increase and vaginitis for the nurses. I had more time to focus on the challenging cases and my panel pushed 3000."

Physician:

"I work every day of the week to take care of our patients and have no 'days off.' I function as the quarterback with only 28 hours of scheduled patients and each appointment is 30 minutes. The rest of the time is for e-visits, phone visits and paperwork. A good number of these patients are new to our system. They heard about how we deliver care differently and are intrigued! Each of these patients has a complete intake by our staff to update all of their history as well as current questions and needs. We address every issue and take the time to create and document a care plan for each issue including preventive care. We make sure the patient takes ownership for the care plan as well and has access to it."

Registered Nurse:

"I was uncomfortable with the added responsibility at first ... I was adjusting blood pressure medications based on a protocol. I could ask for help, but I spent many hours preparing for the next day at home."

Registered Nurse:

"But two years later, when we had to scale nurse visits back because we couldn't bill for them, we [nurses] went back to being shackled to our phones and computers."

Nurse Practitioner:

"Those who struggled had to let go of what they were used to doing, had to trust that someone else would do it. We had to work as a team." 


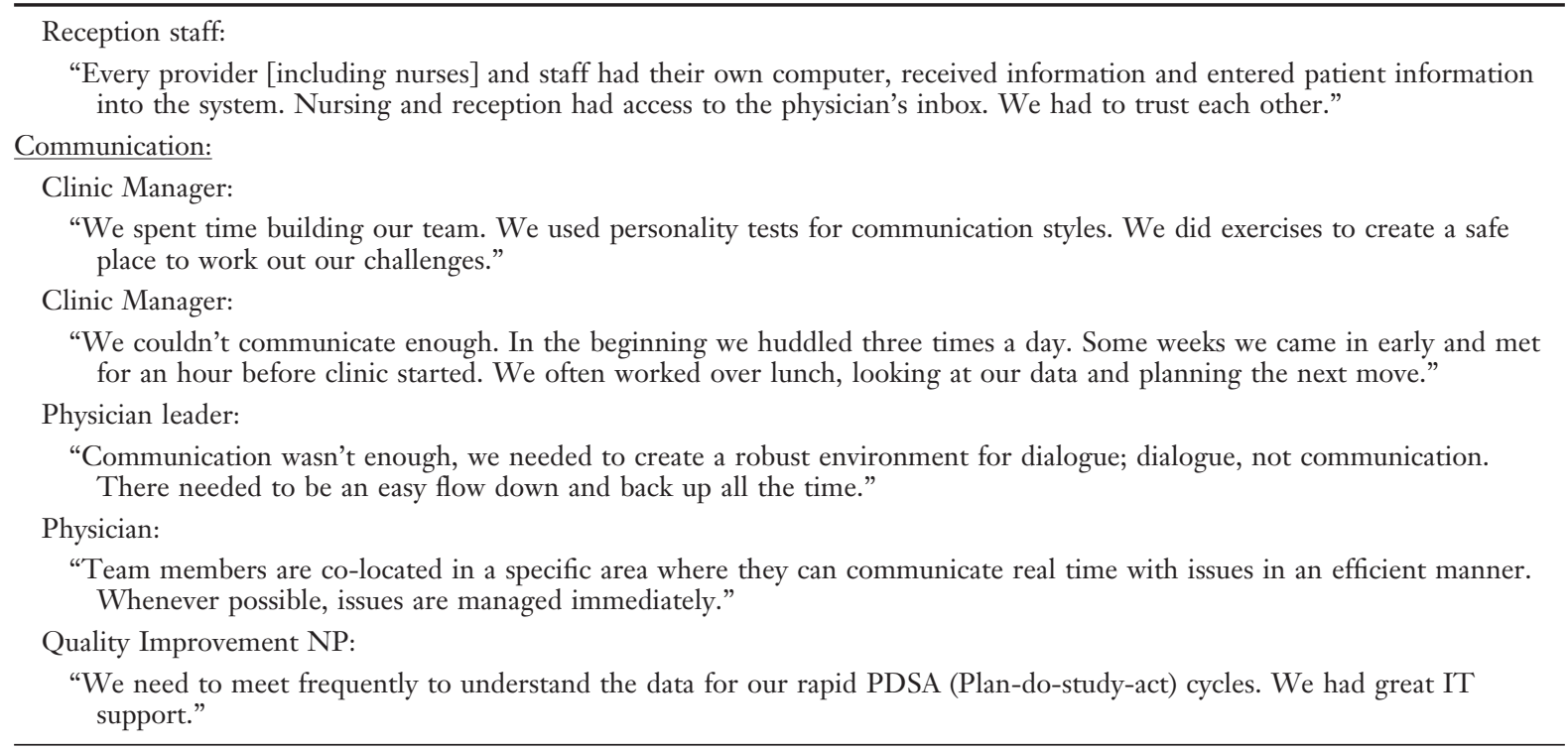

example, in their expanded roles, "nurses started overseeing self-collected samples for vaginitis. $\mathrm{Pa}$ tients celebrated the end of stirrups, but lab personnel were initially reluctant to accept the specimens." Hospital administrators "pushed back" when improved patient management resulted in fewer hospital admissions and emergency department visits.

The personal and professional responses to role redefinition occurred because the patient did not belong to the physician or nurse practitioner, but rather to the clinician and his/her team. A health system leader noted: "Doctors who own their results and cannot share the work with the team still complain about WAC [work after clinic]." Nursing staff talked about the new responsibilities that came with expanded duties and the accompanying stress caused by "homework" on their own time in preparation for the expanded nurse visits. Nurses were working at the top of their licenses more than before, such as following guidelines to direct changes in hypertensive medications or order appropriate labs, employing motivational interviewing skills to coach behavior change, or collecting vaginal specimens. Despite this "stress" they eventually embraced the new challenges and reported disappointment when the nurse visits were scaled back because they did not generate enough revenue in the fee-for-service payment model. Some "felt shackled to their phones and computers" now that they were back to doing only triage.
Many reception staff and medical assistants were cross trained and "most enjoyed the variety and learned to appreciate what each other did." A few reception staff were moved into the pods as "team coordinators." A few medical assistants were trained for new roles such as health coach. "New job descriptions were tweaked overtime."

The role redefinition and new role creation demanded team work. The team-based approach to care required depending and trusting each other. A receptionist noted: "Every provider, nurse, and other staff had their own computer, received information, and entered patient information into the system. Nursing and reception had access to the physician's inbox. We had to trust each other."

The third theme was the importance of communication. Clinics did team-building exercises and invested time to achieve good communication. Eventually they rearranged physical space, colocating physicians and nurses in a pod with a team coordinator (reception staff member). This enhanced "in the moment" communication and prevented patients from waiting for answers to their phoned-in or portal questions.

Team huddles and more frequent meetings allowed for sharing information and "working rapid plan-do-study-act cycles." In the early months huddles took place up to 3 times daily. Additional meetings were scheduled before clinic or over the noon hour. A manager said, "You cannot communicate enough." But 1 physician leader noted that 
Figure 1. Culture assessment scores $^{13}$ (mean score on a 1 to 5 scale with 5 highest) for Clinics A and B compared with the comparison clinics that had not yet transitioned.

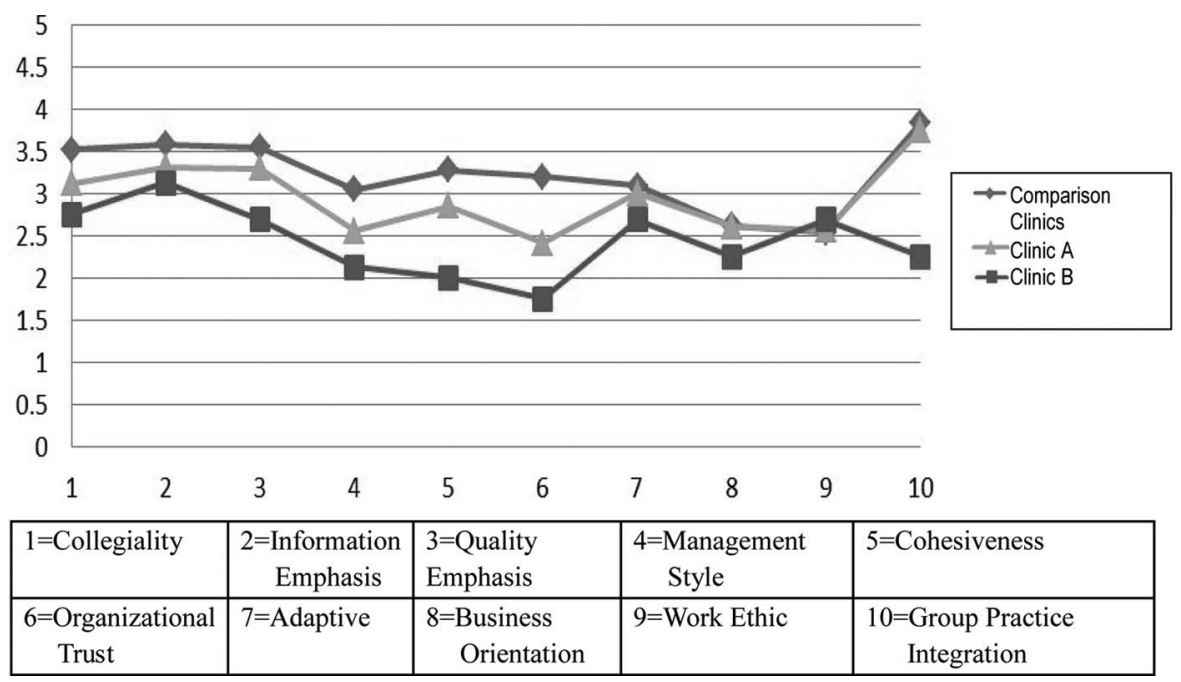

the goal was more than communication, "dialogue [was the goal], an easy flow down and back up all the time." To be successful clinicians, staff and managers problem solved work flows and how to best manage patient care as a team.

Part of the communication included time to celebrate the hard work and show appreciation. A clinic manager explained: "They needed kudos, saying thank you. It was especially important in the beginning."

Figure 1 shows the culture assessment scores for Clinics $\mathrm{A}$ and $\mathrm{B}$ compared with the comparison clinics, 6 primary care clinics owned by another health system in the area that had not yet transitioned to a patient-centered home care model. The comparison clinics had an equal or higher mean scores versus the transition clinics, and, likewise, the first-wave transition clinic (Clinic A) had an equal or higher mean score compared with the second-wave clinic (Clinic B). The 3 groups were most similar on adaptive, business orientation, and work ethic.

Figure 2 shows that patient satisfaction declined during the transition possibly reflecting the reorientation of clinicians and staff, but after a year returned to the earlier rates, then surpassed prior scores, and remained high.

\section{Discussion}

The 2 clinics in our case study transitioned to open-access patient driven models that included elements of the advanced primary care initiatives

Figure 2. Percent of patient respondents rating their health care experience at Clinic A as satisfactory or very satisfactory on Press Ganey patient satisfaction instruments. ${ }^{14} 2007$ was before transition; 2008 and 2009 during transition; and 2010 to 2013 after transition.

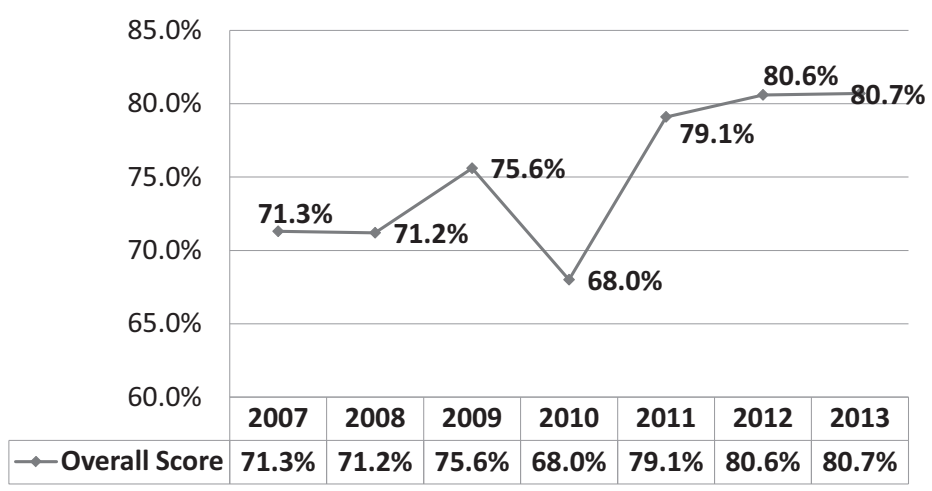


today. ${ }^{16,17}$ In addition, they demonstrated that expanded clinical teams can improve access to care and patient satisfaction while reducing costs. ${ }^{4,5}$ They reorganized how primary care was provided with more patient care managed by nurses, via portal and over the phone. They could not sustain the transformation because the payment model did not change quickly enough. As demonstrated above, this was disappointing to clinicians and staff and may have impacted the culture assessment scores. Other researchers ${ }^{18}$ have found that the fee for service model does not adequately support new care models. Now that health insurers such as Medicare are addressing this payment issue ${ }^{17}$, clinics are incentivized to transition to more efficient and effective models with better value.

The current literature provides some guidance to transforming clinics in terms of identifying the care models, necessary resources, and costs associated with transitions, and how financial costs can be negotiated. ${ }^{2-3,8-12,19-21}$ The limited literature on the human costs largely deals with structural changes and new hires. The toll on clinicians and staff and the responses to those demands have not been well documented. We found that transitions of this magnitude, in these 2 clinics, presented challenges as well as opportunities at all levels of the organization. Setting aside the frustration that occurred because of needing to retrench, many embraced the change. They responded to the new opportunities to address patient needs more fully and efficiently, to expand their own skills, and to work as part of a team. "They were working harder, but enjoying it more."

However, others found it difficult and disruptive and some physicians left because they could not give up their "solo Western hero" identities. The stress levels of nurses were high as they studied on their own time to be competent at expanded duties and took on new responsibilities. For nurses this was practicing at the top of their license and in some cases perhaps beyond, that is, ordering a medication change. Although this was done under physician-nurse constructed protocols and with the final signature of a physician or nurse practitioner, the expanded role was a new expectation and required additional effort and time. Other medical assistants and reception staff were also tasked with new responsibilities. As change theory suggests, it is human nature to have resistance. ${ }^{22}$ Bodenheimer ${ }^{19}$ points out that some physicians struggle with the loss of their central role with the patient and never fully engage in the team model and some medical assistants remain unenthusiastic about their work despite efforts to bring all staff along during the transition.

The transition took more time than expected and the stress related to role adjustments for clinicians and nurses were largely unanticipated or underestimated by the health system leaders who lead the transition. "The letting go of what they were used to doing and trusting someone else to do it" was very difficult for some physicians. Expanding scope of practice was challenging for some nurses. Change leaders need to anticipate and plan for these adjustments with adequate time, training, and support until health profession schools graduate trainees with more experience in new models of team-based care.

Typically, organizational cultures are difficult to change, especially in health care. ${ }^{23}$ The extensive restructuring that took place in these 2 clinics coupled with the turnover and then some retrenching could be expected to influence the cultural dimensions. Unfortunately, we do not have pretransition culture data for comparison. However, if the transition had an influence on the cultures of these practices, it seems that it was largely negative. Respondents gave lower ratings for management style, degree of practice cohesion/integration, and organizational trust. Clinic A was in the first wave of change, was actively involved in crafting the new model with consultants, and sustained the changes for 2 full years before financial viability forced regression. Secondwave Clinic B, restructured rapidly, employed the team-based model that was handed to them with some latitude to innovate. As a result, they did not own the changes in the same way that Clinic A did. Then after 1 year they had to retrench. The lesson learned here may be that ownership of the change makes the changes more palatable and a sustainable payment model is imperative.

Finally, as others have reported, patient satisfaction improves with the patient-centered focus. ${ }^{21,24,25}$ Many patients, especially those with complex medical and social needs, may find the patient-centered team approach more convenient and efficient. Those who do not will likely vote with their feet as long as they are able.

This case study has the limitations associated with the acquisition and interpretation of qualitative data from samples of modest size and collected retrospectively. In addition, while interview information was carefully vetted, we cannot assure that 
other clinics with different cultures and structures would achieve similar results. Clinicians and staff who left were not available to us. Data provided by Clinic B were incomplete.

Despite these limitations, our work confirms what other studies have demonstrated: creating a patient-centered, high-quality, primary-care-teambased delivery model is hard work with real human costs that should be anticipated. Change takes an emotional toll and demands extensive role redefinition and training along with the preparation to take on new duties. While this is stressful, the advanced primary care models offer opportunities for health professionals to grow. Health profession education must prepare students for these new roles. In the meantime, leadership leading care model transformation should anticipate and plan to manage the emotional toll, provide enough time, education, and support for clinicians, nurses, and staff as they wrestle with stress, fear, insecurity, and uncertainty of their redefined roles. Clinicians and staff should be given as much ownership of the changes as possible. Finally, incorporating knowledge and experience with teambased, interprofessional care during training is essential to properly prepare all health professionals for future practice.

To see this article online, please go to: http://jabfm.org/content/ 30/1/16.full.

\section{References}

1. Berwick DM, Nolan TW, Whittington J. The triple aim: care, health, and cost. Health Affairs 2008;27: $759-69$.

2. McNellis RJ, Genevro JL, Meyers DS. Lessons learned from the study of primary care transformation. Ann Fam Med 2013;11:S1-S5.

3. Grumbach K, Grundy P. Outcomes of implementing patient-centered medical home interventions. Washington, DC. Patient-Centered Primary Care Collaborative; 2010.

4. Kralewski J. Transition of primary care medical group practices to next generation models. Publication: 150054-5-EF Content last updated May 2015. Agency for Healthcare Research and Quality, Rockville, MD. Available from: http://www.ahrq.gov/professionals/ systems/primary-care/pcp-costs/kralewski-costs.html. Accessed July 23, 2016.

5. Kralewski J, Zink T, Dowd, B, Tong J. A tale of two family practice clinics: How they adopted patientcentered care, but couldn't sustain it. Physician Leadership Journal March-April 2016. Available from: http://www.physicianleaders.org/news/plj- articles/2016-march-april/2016/03/03/a-tale-oftwo-family-practice-clinics. Accessed July 21, 2016.

6. Yin RK. Case study research: Design and methods. Newbury Park, CA: Sage Publications, 1989.

7. Kralewski JE, Dowd BE, Cole-Adeniyi T, Gans D, Malakar L, Elson B. Factors influencing physician use of clinical electronic information technologies after adoption by their medical group practices. Health Care Manage Rev 2008;33:361-7.

8. Solberg LI, Crain AL, Tillema JO, et al. Challenges of medical home transformation reported by 118 patient-centered medical home (PCMH) leaders. J Am Board Fam Med 2014;27:449-57.

9. Homer CJ, Baron RJ. How to scale up primary care transformation: What we know and what we need to know? J Gen Intern Med 2010;25:625-9.

10. Taylor EF, Machta RM, Meyers DS, Genevro J, Peikes DN. Enhancing the primary care team to provide redesigned care: The roles of practice facilitators and care managers. Ann Fam Med 2013;11:80-3.

11. Gill JM, Bagley B. Practice transformation? Opportunities and costs for primary care practices. Ann Fam Med 2013;11:202-5.

12. Peikes DN, Reid RH, Day TJ, et al. Staffing patterns of primary care practices in the comprehensive primary care initiative. Ann Fam Med 2014; 142-9.

13. Kralewski JE, Wingert TD, Barbouche MH. Assessing the culture of medical group practices. Med Care 1996;34:377-88.

14. Press Ganey Patient Experience Instruments. Available from: http://www.pressganey.com/solutions/ patient-experience/survey-data-collection. Accessed August 11, 2016.

15. Borkan J. Immersion/crystallization. In: Crabtree BF, Miller M, eds. Doing qualitative research. 2nd ed. Thousand Oaks, CA: Sage; 1999, 179-94.

16. Rosenthal TC. Are we learning more about patientcentered medical homes (PCMHS), or learning more about primary care? J Am Board Fam Med 2016;29:4-7.

17. Comprehensive Primary Care Plus. Centers for Medicare \& Medicaid Services. Baltimore, MD. Available at: https://innovation.cms.gov/initiatives/comprehensiveprimary-care-plus. Accessed July 21, 2016.

18. Crabtree BF, Nutting PA, Miller WL, Stange KC, Steward EE, Jaen CR. summary of the national demonstration project and recommendations for the patient-centered medical home. Ann Fam Med May 1, 2010;8(Suppl 1):S80-S90.

19. Bodenheimer T, Willard-Grace R. Teamlets in primary care: Enhancing the patient and clinician experience. J Am Board Fam Med 2016;29: 135-8.

20. Bodenheimer T, Ghoroh A, Willard-Grace R, Grumbach K. The 10 building blocks of highperforming primary care. Ann Fam Med 2014; $166-17$. 
21. Jackson GL, Powers BJ, Chatterjee R, et al. Improving patient care. The patient centered medical home. A Systematic Review. Ann Intern Med 2013;158:169-78.

22. Kotter JP. Leading change: Why transformation efforts fail. Harvard Business Review 2007;1:4.

23. Davies HT, Nutley SM, Mannion R. Organisational culture and quality of health care. Quality in Health Care 2000;9:111-9.
24. Solberg LI, Asche SE, Fontaine P, Flottemesch TJ, Anderson LH. Trends in quality during medical home transformation. Ann Fam Med 2011;9: 515-21.

25. Day J, Scammon DL, Kim J, et al. Quality, satisfaction, and financial efficiency associated with elements of primary care practice transformation: preliminary findings. Ann Fam Med 2013;11(Suppl 1): S50-S59. 